ОСОБЕННОСТИ ПСИХОЭМОЦИОНАЛЬНОГО СОСТОЯНИЯ, ПИЩЕВОГО ПОВЕДЕНИЯ И ПОКАЗАТЕЛЕЙ ГОРМОНАЛЬНО-АДИПОКИНОВОЙ РЕГУЛЯЦИИ МЕТАБОЛИЗМА У МУЖЧИН С ПОДКОЖНЫМ И АБДОМИНАЛЬНЫМ ТИПАМИ РАСПРЕДЕЛЕНИЯ ЖИРА

(с) И.Г. Мохова* , Б.Б. Пинхасов, Н.И. Шилина, С.В. Янковская, В.Г. Селятицкая

Федеральный исследовательский центр фундаментальной и трансляционной медицины, Новосибирск, Россия

Обоснование. Развитие ожирения и ассоциированных с ним нарушений здоровья прямо связано с нарушениями пищевого поведения и психоэмоционального состояния. Представляет значительный интерес оценка особенностей этих показателей у мужчин с подкожным и абдоминальным типами распределения жира, которые ассоциированы с различными гормональными и адипокиновыми механизмами, влияющими не только на параметры метаболизма, но и на пищевое поведение.

Цель. Изучить особенности психоэмоционального состояния, пищевого поведения и их связи с показателями гормонально-адипокинового статуса у мужчин с разной топографией распределения жировой ткани.

Методы. Проведено одноцентровое, поперечное выборочное обследование 99 мужчин в возрасте от 27 до 68 лет. Выполнено антропометрическое обследование, на основании которого были сформированы 4 группы мужчин. Группу 1 (сравнения) составили мужчины с нормальной массой тела. Группу 2 составили мужчины с избыточной массой тела, ожирением I степени и нижним подкожным типом распределения жира (ПТРж). Группу 3 составили мужчины с избыточной массой тела, ожирением I степени и центральным абдоминальным типом распределения жира (АТРж); группу 4 - мужчины с ожирением 2 и 3 степени с АTPЖ. С использованием опросника DEBQ определены типы пищевого поведения, с использованием Госпитальной шкалы тревоги и депрессии - выраженность тревожно-депрессивных нарушений; для оценки степени нарушения удовлетворенности образом тела использовали тест-опросник «Исследования образа тела». Проведено исследование сыворотки крови на содержание глюкозы, триглицеридов, инсулина, лептина и адипонектина.

Результаты. Показано, что для мужчин с АТРЖ по сравнению смужчинами с ПТРЖ характерны выраженные нарушения показателей углеводно-жирового обмена и его регуляции: гипергликемия, гипертриглицеридемия, гиперинсулинемия и инсулинорезистентность, гиперлептинемия и гипоадипонектинемия. Мужчинам с АТРЖ свойственны экстернальный тип пищевого поведения, повышенный уровень депрессивных нарушений и недовольство образом собственного тела, в то время как мужчинам с ПтРЖ - эмоциогенный тип пищевого поведения и удовлетворение образом собственного тела. Сравнительный анализ величин показателей между мужчинами групп 3 и 4 с АТРж показал, что в группе 4 у мужчин на фоне большего количества массы жировой ткани усиливаются гиперлептинемия и инсулинорезистентность, при этом выраженность нарушений пищевого поведения, тревоги и депрессии не различаются между группами.

Заключение. Топография распределения жировой ткани ассоциирована с психофизиологическими, метаболическими и гормонально-адипокиновыми особенностями, лежащими в основе развития первичного ожирения у мужчин.

КЛЮЧЕВЫЕ СЛОВА: ожирение; подкожный тип распределения жира; абдоминальный тип распределения жира; пищевое поведение; инсулин; лептин; адипонектин.

\title{
THE FEATURES OF PSYCHOLOGICAL STATE, EATING BEHAVIOR, HORMONAL AND ADIPOKINE REGULATION OF METABOLISM IN MEN WITH SUBCUTANEOUS AND ABDOMINAL FAT DISTRIBUTION
}

\author{
(c) Iraida G. Mokhova*, Boris B. Pinkhasov, Nadejda I. Shilina, Svetlana V. Yankovskaya, Vera G. Selyatitskaya
}

Federal Research Center for Fundamental and Translational Medicine, Novosibirsk, Russia

BACKGROUND: development of obesity and obesity related conditions are directly associated with eating disorders and psychological state. There is increasing focus on the assessment the characteristics of these indicators in men with subcutaneous and central fat distribution, which are associated with various hormonal and adipokine mechanisms that effect on parameters of metabolism and eating behavior.

AIMS: to study the characteristics of the psychologycal state, eating behavior and their relationship with hormonal and adipokine status in men with different fat distribution.

MATERIALS AND METHODS: a single-center, cross-sectional study of 99 men aged 27 to 68 years was performed. 4 groups of men were formed after anthropometric examination. Group 1 (comparison) consisted of men with normal body weight. Group 2 consisted of overweight men, class I obesity and lower subcutaneous fat distribution (SFD). Group 3 consisted of overweight men, men with class I obesity and abdominal fat distribution (AFD); group 4 - men with class II obesity and 
class III obesity AFD. Eating behavior were determined with DEBQ questionnaire, severity of anxiety-depressive disorders were investigated with the Hospital Anxiety and Depression Scale; body image satisfaction was assessed with body image questionnaire. Serum glucose, triglycerides, insulin, leptin and adiponectin were estimated.

RESULTS: it was shown, that men with AFD had severe metabolic disorders: hyperglycemia, hypertriglyceridemia, hyperinsulinemia and insulin resistance, hyperleptinemia and hypoadiponectinemia, than men with SFD. Men with AFD had an external type of eating behavior, with sever expressed depressive disorders and body image dissatisfaction. Men with SFD had an emotional type of eating behavior and higher body image satisfaction. A comparative analysis between men from 3 and 4 groups with AFD showed that in group 4 in men had higher hyperleptinemia and insulin resistance and there are no differences in severity of eating disorders, anxiety and depression between the groups.

CONCLUSIONS: adipose tissue topography is associated with the psychophysiological, metabolic, hormonal and adipokine characteristics that underlie the development of primary obesity in men.

KEYWORDS: obesity; subcutaneous fat; abdominal fat; eating behavior; insulin; leptin; adiponectin.

\section{ОБОСНОВАНИЕ}

В настоящее время ожирение является глобальной медико-социальной проблемой развитых и развивающихся стран и носит характер пандемии [1, 2]. Ожирение повышает риски развития сахарного диабета 2 типа, сердечно-сосудистых заболеваний и ассоциированных с ними клинических состояний [3]. Среди причин развития первичного ожирения важными являются изменение образа жизни; гиподинамия; нарушения питания, включая увеличение объема потребляемой пищи из-за привлекательности вкуса и запаха; реклама и потребление пищевых продуктов с высоким содержанием жиров, углеводов, и т.Д. [4]. Все это формирует и закрепляет нарушения пищевого поведения, которые, с одной стороны, ассоциированы с психоэмоциональными реакциями, тревогой и вызывают развитие депрессии, а с другой - со стойкими гормонально-метаболическими нарушениями. Показано, что развитие ожирения и ассоциированных с ним нарушений здоровья прямо связано с изменениями пищевого поведения и психоэмоционального состояния $[5,6]$.

Первичное ожирение может сопровождаться преимущественным накоплением жировой ткани в подкожных депо или в висцеральных депо в абдоминальной области. Гетерогенность ожирения опосредована анатомической, морфологической и функциональной гетерогенностью жировой ткани [7]. В норме масса висцерального жира составляет 10-20\% у мужчин и 5-8\% у женщин от общего количества жира в организме, а основная часть жира - около 80\% - представлена подкожным жиром, сосредоточенным в основном в абдоминальном и глютеофеморальном депо [8]. Адипоциты подкожного жира, также как и висцерального, выполняют важную физиологическую и регуляторную роль. Жировая клетчатка принимает участие в энергетическом обмене и терморегуляции, поддержании метаболического, иммунного, адаптивного и репродуктивного статусов организма [7]. В последние годы большим шагом к пониманию патофизиологических механизмов формирования ожирения стало выделение фенотипов ожирения: метаболически здорового и метаболически нездорового [9]. В основу выделения данных типов положено топографическое доминирование подкожной или висцеральной жировой ткани, которые существенно различаются по метаболической активности $[10,11]$ и спектру синтезируемых гормонов и адипокинов [7]. Так, один из ключевых адипокинов жировой ткани - адипонектин обладает противовоспалительным, антиатерогенным и кардиопротек- тивным действием и в большей степени секретируется в висцеральной жировой ткани, однако его уровень при ожирении снижается [12]. Другой адипокин - лептин синтезируется преимущественно в подкожной жировой ткани и участвует в регуляции пищевого поведения [5, 13].

Накопление жира в абдоминальной области связано с развитием висцерального ожирения, которое, в свою очередь, выступает независимым фактором риска сердечно-сосудистых заболеваний, инсулинорезистентности, сахарного диабета 2 типа, атеросклероза и других состояний, ускоряющих процессы старения организма [14, 15]. Накопление подкожного жира в глютеофеморальной области рассматривают преимущественно с позиций решения косметических проблем, поскольку на начальных стадиях накопления избыточной массы жировой ткани выраженных нарушений углеводно-жирового обмена нет и, соответственно, риск сердечно-сосудистых заболеваний минимален [16].

Если с позиций гормонально-метаболических нарушений разница между подкожным и абдоминальным типами распределения жира определена [7], то сведений об особенностях пищевого поведения и психоэмоциональных реакциях у лиц с этими разными типами распределения жира крайне мало. Особенно это касается лиц мужского пола, у которых в зрелом возрасте частота встречаемости подкожного типа распределения жира меньше, чем абдоминального, в силу чего его редко выделяют как отдельный тип распределения жира у зрелых мужчин.

\section{ЦЕЛЬ}

Изучить особенности психоэмоционального состояния и пищевого поведения в их взаимосвязи с показателями гормонально-адипокинового статуса у мужчин с разной топографией распределения жировой ткани.

\section{МЕТОДЫ}

\section{Дизайн исследования}

Проведено обсервационное одноцентровое поперечное выборочное неконтролируемое исследование.

\section{Критерии соответствия}

Критерии включения: мужской пол, избыточная масса тела и/или первичное ожирение алиментарно-конституциональной природы.

Критерии исключения: вторичное ожирение. 


\section{Условия проведения}

В исследовании приняли участие мужчины, проходившие обследование и лечение в терапевтической клинике Федерального исследовательского центра фундаментальной и трансляционной медицины (Новосибирск).

\section{Продолжительность исследования}

Набор материала продолжался с сентября 2018 по декабрь 2018 г. Образцы сывороток исследовали одномоментно после завершения сбора всего биологического материала.

\section{Описание медицинского вмешательства}

Всем пациентам проводили общеклиническое обследование, антропометрическое обследование, включающее измерение длины тела (ДТ), массы тела (МТ), окружностей талии (ОТ) и бедер (ОБ). Относительное (Жир, \%) и абсолютное содержание жировой ткани в организме (Жир, кг) измеряли методом импедансометрии. В сыворотке крови, взятой в утреннее время натощак, определяли биохимические показатели углеводно-жирового обмена, гормоны и адипокины; с помощью опросников оценивали пищевое поведение, выраженность тревоги, депрессии и отношение к образу собственного тела.

\section{Основной исход исследования}

Определены и изучены антропометрические показатели, характеризующие количество и топографию распределения жировой ткани, необходимые для выделения степени и типа ожирения; гормонально-метаболические показатели, характеризующие состояние углеводно-жирового обмена; типы нарушений пищевого поведения и показатели, характеризующие психоэмоциональное состояние пациентов в исследуемых группах.

\section{Анализ в подгруппах}

Группу 1 (сравнения) составили мужчины с нормальной массой тела; группу 2 - мужчины с избыточной массой тела, ожирением и нижним подкожным типом распределения жира (ПТРЖ) группу 3 - мужчины с ожирением 1 степени и центральным абдоминальным типом распределения жира (АТРЖ); группу 4 - мужчины с ожирением 2 и 3 степеней и центральным АТРЖ.

\section{Методы регистрации исходов}

На основании отношения ОТ/ОБ выделяли тип ожирения. При величине отношения ОТ/ОБ <0,95 у.е. выделяли пациентов с подкожным типом распределения жира, при величине ОТ/ОБ >0,95 у.е., - пациентов с абдоминальным типом распределения жира. Импедансометрия выполнялась с применением прибора фирмы OMRON (Япония). Для определения типа пищевого поведения (ПП) и оценки его выраженности в баллах использовали опросник DEBQ, включающий четыре независимые анкеты. По числу баллов в анкете также выявляли клинически значимые случаи нарушения ПП. За граничные значения, превышение которых указывало на наличие клинически значимого нарушения ПП, принимали следующие величины в баллах: для эмоциогенного - 2,03; компульсивного - 2,60; экстернального - 2,68; ограничительного 2,43 балла [17]. Выраженность тревожно-депрессивных нарушений выявляли с помощью «Госпитальной шкалы тревоги и депрессии» (HADS). При интерпретации результатов учитывали суммарный показатель по каждой подшкале. Сумму баллов расценивали как: 0-7 - норма (отсутствие достоверно выраженных симптомов тревоги/депрессии), 8-10 - субклинически выраженная тревога/депрессия, 11 и выше - клинически выраженная тревога/депрессия [18]. Тест-опросник «Исследование образа тела» (ИОТ) использовали для оценки степени нарушения удовлетворенности образом тела [19]. Результаты тестирования интерпретировали следующим образом: при сумме 10 баллов и меньше оценивали как позитивное отношение пациента к собственной внешности, которое не влияет негативно на его повседневную жизнь. Сумму баллов от 11 до 20 оценивали как удовлетворительное отношение пациента к собственной внешности большую часть времени, однако в определенных ситуациях образ тела, а следовательно, и уверенность в себе, могут колебаться, в результате существует риск появления чувства тревоги в отношении собственного тела, его размеров, формы и внешнего вида. Сумму баллов более 21 интерпретировали как дискомфорт, при котором воспринимаемый внешний облик испытуемого доставляет ему массу психологических проблем.

Биохимическое исследование включало определение содержания в сыворотке крови утром натощак глюкозы и триглицеридов с использованием коммерческих наборов фирмы BioCon (Германия). Иммуноферментным методом с использованием коммерческих наборов в сыворотке крови измеряли содержание иммунореактивного инсулина (ИРИ) (Monobind Inc., США; нормативные величины 0,7-9,0 мкЕд/мл), лептина (Diagnostic Biochem, Канада; нормативные величины 3,7-11,1 нг/мл), адипонектина (Assay Max Human Adiponectin Elisa Kit, Assaypro, США; нормативные величины 8,3-13,9 мкг/мл). Рассчитывали индекс инсулинорезистентности HOMA-IR по формуле: ИРИ (мкЕд/мл)×глюкоза плазмы натощак (ммоль/л)/22,5.

\section{Этическая экспертиза}

Проведение исследования было одобрено локальным Комитетом по биомедицинской этике Федерального исследовательского центра фундаментальной и трансляционной медицины (заключение № 13-1, от 25.06.2018). С пациентами проводили беседу, объясняющую цель и задачи исследования; от них было получено информированное согласие на участие в исследовании.

\section{Статистический анализ}

Принципы расчета размера выборки: предварительно не рассчитывалась.

Методы статистического анализа данных: статистическую обработку данных проводили с использованием программы Statistica 10 (StatSoft, США). Результаты в таблицах и тексте указывали как среднее арифметическое \pm среднеквадратичное отклонение $(\mathrm{M} \pm \mathrm{SD})$ или \%. Для множественных сравнений использовали непараметрический критерий Крускала-Уоллиса и критерий $\mathrm{X}^{2}$ Пирсона с поправкой Йейтса; для парных сравнений критерий Манна-Уитни; для характеристики зависимости параметров применяли коэффициент ранговой корреляции Спирмена (r). Минимальную вероятность справедливости нулевой гипотезы принимали при 5\% уровне значимости $(p<0,05)$. 


\section{РЕЗУЛЬТАТЬ}

Объекты (участники) исследования

Анализ возрастной и антропометрических характеристик мужчин в выделенных группах представлен в таблице 1. Динамика увеличения возраста мужчин от группы 2 к группе 3 и далее к группе 4 согласуется с ранее полученными результатами [20], указывающими, что в ходе онтогенеза частота ПтРж снижается и в старших возрастных группах доминирует АТРЖ. Мужчины группы 2 с ПТРЖ по всем антропометрическим характеристикам, кроме величины ОТ/ОБ, отличались в большую сторону от мужчин группы 1 с нормальной массой тела. Отличия величин всех антропометрических показателей у мужчин групп 3 и 4 с АТРЖ от соответствующих значений у мужчин группы 1 были выражены в еще большей степени. Мужчины из группы 4 отличались от мужчин из группы 3 по всем антропометрическим показателям в сторону повышения их величин за исключением равной величины отношения ОТ/ОБ.

Мужчины групп 2 и 3 не различались по величинам антропометрических характеристик, кроме ОТ и, соответственно, величины отношения ОТ/ОБ, значение которого служило критерием выделения этих групп. Поскольку возраст, количество жировой ткани и выраженность ожирения у мужчин в группах 2 и 3 не различались, срав- нительный анализ гормонально-метаболических и психоэмоциональных показателей позволил выявить особенности, характерные именно для ПТРЖ и АТРЖ.

\section{Основные результаты исследования}

Содержание триглицеридов в сыворотке крови обследованных мужчин повышалось в ряду от группы 1 к группе 4 (табл. 2), что, очевидно, связано с ростом относительного и абсолютного содержания жировой ткани в организме (см. табл. 1.). При этом разницы в содержании триглицеридов в сыворотке крови мужчин 2-й и 3-й групп, как и в содержании жировой ткани, обнаружено не было. В свою очередь, увеличение количества жировой ткани было ассоциировано с выраженным повышением содержания в сыворотке крови лептина - пептидного гормона жировой ткани, принимающего участие в регуляции аппетита и потреблении пищи, расходовании энергии и регуляции массы тела [21, 22]. У мужчин группы 2 уровень глюкозы крови не отличался от величины показателя у мужчин с нормальной массой тела, группы 3 - отмечена тенденция к повышению, а группы 4 - уровень глюкозы был достоверно выше. В ряду от группы 1 к группе 4 отмечено повышение уровня инсулина и величины индекса инсулинорезистентности HOMA-IR (см. табл. 2). Содержание

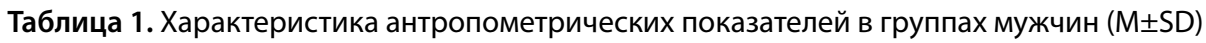

\begin{tabular}{|c|c|c|c|c|c|c|c|c|c|c|}
\hline Показатель & Группа 1 & Группа 2 & Группа 3 & Группа 4 & & & & & & \\
\hline $\mathbf{n}$ & 15 & 26 & 31 & 27 & $1-2$ & $1-3$ & $1-4$ & $2-3$ & $2-4$ & $3-4$ \\
\hline Возраст, лет & $48,4 \pm 11,5$ & $44,7 \pm 10,9$ & $48,4 \pm 8,2$ & $52,1 \pm 9,6$ & - & - & - & - & 0,018 & - \\
\hline 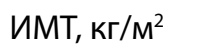 & $23,6 \pm 1,5$ & $29,9 \pm 3,0$ & $31,1 \pm 2,8$ & $39,7 \pm 4,2$ & 0,000 & 0,000 & 0,000 & - & 0,000 & 0,000 \\
\hline Жир, \% & $21,4 \pm 6,6$ & $29,2 \pm 5,6$ & $29,3 \pm 4,9$ & $36,2 \pm 5,2$ & 0,001 & 0,002 & 0,000 & - & 0,000 & 0,000 \\
\hline Жир, кг & $16,4 \pm 6,3$ & $27,1 \pm 7,2$ & $28,5 \pm 6,5$ & $43,2 \pm 10,9$ & 0,000 & 0,000 & 0,000 & - & 0,000 & 0,000 \\
\hline OT, $\mathrm{CM}$ & $87,0 \pm 9,4$ & $95,3 \pm 7,5$ & $108,1 \pm 6,7$ & $120,6 \pm 10,3$ & 0,004 & 0,000 & 0,000 & 0,000 & 0,000 & 0,000 \\
\hline ОБ, см & $92,9 \pm 6,3$ & $102,5 \pm 6,1$ & $100,5 \pm 6,5$ & $111,9 \pm 11,1$ & 0,000 & 0,001 & 0,000 & - & 0,001 & 0,000 \\
\hline OT/OБ, у.е. & $0,94 \pm 0,07$ & $0,93 \pm 0,05$ & $1,08 \pm 0,04$ & $1,08 \pm 0,05$ & - & 0,000 & 0,000 & 0,000 & 0,000 & - \\
\hline
\end{tabular}

Примечание. ИМТ - индекс массы тела, ОТ - окружность талии, ОБ - окружность бедер, ОТ/ОБ - отношение окружности талии к окружности бедер. Данные представлены в виде среднего (M) и стандартного отклонения (SD). Статистически значимыми считались результаты при вероятности справедливости нулевой гипотезы менее 5\% уровня значимости.

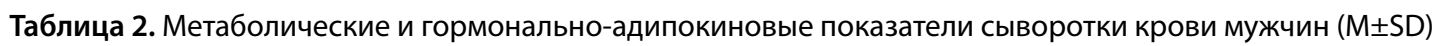

\begin{tabular}{|c|c|c|c|c|c|c|c|c|c|c|}
\hline Показатель & Группа 1 & Группа 2 & Группа 3 & Группа 4 & \multicolumn{6}{|c|}{$\mathbf{p}$} \\
\hline $\mathbf{n}$ & 15 & 26 & 31 & 27 & $1-2$ & $1-3$ & $1-4$ & $2-3$ & $2-4$ & $3-4$ \\
\hline Триглицериды, ммоль/л & $1,4 \pm 0,5$ & $1,8 \pm 0,9$ & $2,1 \pm 0,9$ & $2,8 \pm 1,8$ & - & 0,008 & 0,001 & - & 0,009 & - \\
\hline Лептин, нг/мл & $5,5 \pm 2,1$ & $10,3 \pm 4,4$ & $11,0 \pm 5,9$ & $25,8 \pm 17,3$ & 0,006 & 0,000 & 0,000 & - & 0,000 & 0,002 \\
\hline Глюкоза, ммоль/л & $5,1 \pm 1,0$ & $4,8 \pm 0,6$ & $5,6 \pm 1,4$ & $6,1 \pm 1,6$ & - & - & 0,012 & 0,045 & 0,000 & - \\
\hline Инсулин, мкЕд/мл & $5,3 \pm 4,1$ & $8,3 \pm 4,6$ & $12,7 \pm 9,5$ & $18,2 \pm 13,0$ & - & 0,002 & 0,003 & 0,002 & 0,001 & - \\
\hline HOMA-IR, y.e. & $1,1 \pm 0,9$ & $1,9 \pm 1,2$ & $3,6 \pm 1,8$ & $4,9 \pm 1,5$ & - & 0,001 & 0,001 & 0,001 & 0,001 & - \\
\hline Адипонектин, мкг/мл & $11,6 \pm 8,8$ & $9,2 \pm 5,9$ & $7,4 \pm 3,7$ & $6,4 \pm 3,7$ & - & 0,002 & 0,002 & 0,007 & 0,005 & 0,049 \\
\hline $\begin{array}{l}\text { Лептин/Адипонектин, } \\
\text { у.е. }\end{array}$ & $0,6 \pm 0,3$ & $1,2 \pm 0,7$ & $1,9 \pm 1,6$ & $4,7 \pm 3,9$ & 0,001 & 0,000 & 0,000 & 0,043 & 0,000 & 0,000 \\
\hline
\end{tabular}

Примечание. HOMA-IR (Homeostasis Model Assessment of Insulin Resistance) - индекс инсулинорезистентности. Данные представлены в виде среднего (M) и стандартного отклонения (SD). Статистически значимыми считались результаты при вероятности справедливости нулевой гипотезы менее $5 \%$ уровня значимости. 
в сыворотке крови адипонектина, пептидного гормона с антиапоптотическим, противовоспалительным и антифибротическим эффектами, повышающего чувствительность тканей к инсулину [23], понижалось у мужчин от группы 1 к группе 4. Следует отметить, что по величинам показателей углеводного обмена и его гормональной регуляции, а также адипонектина, мужчины группы 2 не отличались от мужчин группы 1, но статистически значимо отличались от мужчин группы 3.

Расчет отношения величин содержания в сыворотке крови лептина к адипонектину, которое рассматривается как биомаркер раннего обнаружения метаболических нарушений при ожирении, выраженности воспаления в жировой ткани и дисфункции жировой ткани [24], показал девятикратное повышение величины этого индекса от группы 1 к группе 4. Наименьшее повышение величины этого отношения относительно группы 1 отмечено в группе 2, а у мужчин группы 3 величина этого отношения была достоверно выше, чем у мужчин группы 2. При исследовании особенностей пищевого поведения (ПП) было показано, что выраженность проявления всех изученных типов ПП статистически значимо выше у мужчин групп 2, 3 и 4 с избыточной массой тела и ожирением по сравнению с мужчинами из группы 1 с нормальной массой тела (табл. 3). У мужчин группы 2 с ПТРЖ в большей степени выражено эмоциогенное ПП. Количество клинически значимых нарушений эмоциогенного ПП в группах 1, 2, 3 и 4 составило 13,3\%, 38,5\%, 19,4\% и 25,9\% соответственно, однако достоверных различий между группами выявлено не было $\left(x^{2}=4,11, p=0,252\right)$. У мужчин с АТРЖ как в группе 3, так и в группе 4 преобладало экстернальное ПП. Клинически значимые нарушения данного типа ПП встречались в группах 1, 2, 3 и 4 в 13,3\%, $19,2 \%, 41,9 \%$ и 51,9\% случаев $\left(X^{2}=10,03 ; p=0,021\right)$. Частота клинически значимых нарушений ограничительного ПП в группах от 1 к 4 увеличивалась и составила 6,7\%, $19,2 \%, 22,6 \%, 25,9 \%$ случаев $\left(x^{2}=2,38 ; p=0,511\right)$ соответственно. Встречались и сочетанные нарушения ПП. Так, количество клинически значимых сочетаний нарушений ПП в группах 1, 2, 3 и 4 составило 0\%, 19,2\%, 22,6\% и 37,0\% ( $\left.\chi^{2}=7,85 ; p=0,505\right)$ соответственно. Признаки тревоги были выражены у мужчин всех групп в одинаковой мере, но признаки депрессии были выражены в значительно большей степени у мужчин с избыточ- ной массой тела и ожирением относительно мужчин с нормальной массой тела с достоверным повышением выраженности признаков депрессии у мужчин группы 4 относительно группы 2 (см. табл. 3).

Учитывая тот факт, что ПП и психоэмоциональное состояние у мужчин в группах 3 и 4 не различались между собой, анализ корреляционных связей проводили в объединенной группе мужчин с АТРЖ и отдельно в группе с ПТРЖ. Показано, что уровень тревоги у мужчин с ПТРЖ из группы 2 положительно коррелировал с выраженностью эмоциогенного $(r=0,44, p=0,038)$ и компульсивного типов ПП $(r=0,42, p=0,042)$, а у мужчин с АТРЖ уровень депрессии в большей степени коррелировал с выраженностью экстернального ПП ( $r=0,30$, $\mathrm{p}=0,029)$. Психоэмоциональные нарушения у пациентов с ПТРЖ и АТРЖ отразились и на самооценке своей внешности, проведенной с использованием теста-опросника ИОТ. Пациенты, недовольные образом собственного тела: его размерами, формами и т.д., превалировали в группах 3 и 4. Процент таких мужчин в группах 1, 2, 3 и 4 составил 6,7\%, 0,0\%, 16,1\% и 40,7\% ( $x^{2}=14,69$; $\mathrm{p}=0,003)$; в то же время процент лиц, у которых отношение к собственной внешности практически не влияло на их повседневную жизнь, составил 6,7\%, 15,3\%, 35,4\% и 22,0\% ( $\left.x^{2}=5,96 ; p=0,172\right)$ соответственно. Процент лиц, которые в основном чувствовали себя комфортно по отношению к собственному телу в группах 1, 2, 3 и 4 составил $86,6 \%, 84,7 \%, 48,5 \%$ и $37,3 \%\left(x^{2}=18,77 ; p<0,001\right)$ соответственно. Полученные результаты демонстрируют, что мужчины с ПТРЖ практически не испытывают дискомфорта, связанного с накоплением жира, в то время как увеличение количества жировой ткани в абдоминальной области в мужчин с АТРЖ вызывает негативное отношение к образу собственного тела.

Корреляционный анализ взаимосвязей выраженности типов ПП с показателями гормональной и адипокиновой регуляции показал, что у мужчин с ПТРЖ выраженность эмоциогенного ПП была прямо связана с уровнем лептина ( $r=0,41 ; p=0,041)$, а у мужчин с АТРЖ экстернальный тип пищевого поведения был прямо связан с уровнем инсулина $(r=0,25 ; p=0,044)$ и обратно - с уровнем адипонектина $(r=-0,22 ; p=0,080)$.

Нежелательные явления

Отсутствовали.

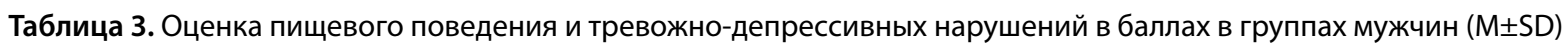

\begin{tabular}{|c|c|c|c|c|c|c|c|c|c|c|}
\hline Показатель & Группа 1 & Группа 2 & Группа 3 & Группа 4 & & & I & & & \\
\hline $\mathbf{n}$ & 15 & 26 & 31 & 27 & $1-2$ & $1-3$ & $1-4$ & $2-3$ & $2-4$ & 3-4 \\
\hline Эмоциогенное ПП, балл & $0,4 \pm 0,2$ & $1,8 \pm 0,7$ & $1,4 \pm 0,4$ & $1,4 \pm 0,4$ & 0,000 & 0,000 & 0,000 & 0,010 & 0,015 & - \\
\hline Компульсивное ПП, балл & $0,8 \pm 0,6$ & $1,2 \pm 0,4$ & $1,2 \pm 0,8$ & $1,1 \pm 0,8$ & 0,015 & 0,095 & 0,015 & - & - & - \\
\hline Экстернальное ПП, балл & $1,7 \pm 0,4$ & $2,0 \pm 0,5$ & $2,4 \pm 0,6$ & $2,5 \pm 0,6$ & 0,015 & 0,000 & 0,000 & 0,045 & 0,020 & - \\
\hline Ограничительное ПП, балл & $0,8 \pm 0,62$ & $1,3 \pm 0,6$ & $1,4 \pm 0,7$ & $1,4 \pm 0,7$ & 0,015 & 0,005 & 0,005 & - & - & - \\
\hline Тревога, балл & $5,7 \pm 3,1$ & $6,5 \pm 5,1$ & $6,6 \pm 3,9$ & $5,7 \pm 2,7$ & - & - & - & - & - & - \\
\hline Депрессия, балл & $2,7 \pm 1,9$ & $4,4 \pm 2,3$ & $5,4 \pm 3,1$ & $5,9 \pm 3,1$ & 0,020 & 0,003 & 0,001 & - & 0,050 & - \\
\hline Тест-опросник ИОТ, балл & $5,6 \pm 4,2$ & $5,9 \pm 4,8$ & $11,8 \pm 10,9$ & $18,6 \pm 11,4$ & - & 0,040 & 0,000 & 0,010 & 0,000 & 0,025 \\
\hline
\end{tabular}

Примечание. ПП - пищевое поведение, ИОТ - исследование образа тела. Данные представлены в виде среднего (М) и стандартного отклонения (SD). Статистически значимыми считались результаты при вероятности справедливости нулевой гипотезы менее 5\% уровня значимости. 


\section{ОБСУЖДЕНИЕ}

Резюме основного результата исследования

Топография распределения жировой ткани у мужчин зрелого возраста ассоциирована не только с метаболическими и гормонально-адипокиновыми характеристиками организма, но и с лежащими в основе развития первичного ожирения нарушениями ПП и депрессией. Для мужчин с АТРЖ, наряду с гипергликемией, гипертриглицеридемией, гиперинсулинемией и инсулинорезистентностью, гиперлептинемией и гипоадипонектинемией, характерны экстернальный тип ПП, повышенный уровень депрессивных нарушений и недовольство образом собственного тела. Для мужчин с ПТРЖ на фоне меньшей выраженности дислипидемии и гиперлептинемии характерны эмоциогенный тип ПП и удовлетворение образом собственного тела.

\section{Обсуждение основного результата исследования}

Тип распределения жировой ткани в организме определяется конституцией человека, возрастом и другими факторами [20]. Обсуждается гипотеза, что за общую эпидемию ожирения в мире отвечают условия окружающей среды, в то время как тип распределения жира в организме определяют генетические факторы [25]. В частности, показано, что различия в экспрессии двух генов НОХС9 и НОХС10 в жировой ткани вносят вклад в ее морфологические и метаболические свойства в разных жировых депо и могут определять индивидуальные различия в распределении жира, степень гипертрофии адипоцитов и увеличение жировых депо [26]. Авторы отмечают, что эти гены входят в число генов, контролирующих процесс развития, и могут определять тип индивидуального распределения жировой ткани у взрослых уже на эмбриональной стадии развития.

Известно, что метаболические нарушения, негативные сдвиги в гормональной и адипокиновой регуляции усиливаются при нарастании степени ожирения [21, 22, 23]. Поэтому для сравнительного изучения ассоциации психофизиологических характеристик организма, а также пищевого поведения, с типом распределения жира в организме были выделены группы мужчин с равной степенью ожирения по показателям ИМТ, абсолютного и относительного количества жира в организме, но разными типами распределения жира - нижний ПТРЖ (группа 2) и центральный АТРЖ (группа 3). Из общего числа обследованных мужчин с избыточной массой тела и ожирением частота лиц с ПТРЖ составила 31\%, что совпадает с ранее полученными сведениями [27]. У всех обследованных мужчин с ожирением 2 и 3 степени по величине отношения ОТ/ОБ был выявлен АТРЖ (группа 4). Можно говорить о том, что при выраженном прогрессировании ожирения нижний тип распределения жира может не выявляться за счет чрезмерного накопления жировой ткани во всех жировых депо, на что указывает значительное увеличение величины не только ОТ, но и ОБ у мужчин группы 4 по сравнению с мужчинами групп 2 и 3.

Проведенное исследование выявило связь ожирения с нарушениями ПП и психоэмоционального состояния в виде тревожно-депрессивного синдрома. Аналогичные сведения получены и другими авторами [5, 19]. Однако для разных типов распределения жира в организме оказались характерны различные варианты нарушений пищевого поведения и психологических особенностей личности. Так, мужчины с АТРЖ относительно мужчин с ПТРЖ чаще имели экстернальный тип нарушений пищевого поведения и более высокий уровень аффективных нарушений, в то время как по уровню тревоги мужчины с АТРЖ и ПТРЖ не различались. В то же время мужчины с ПТРЖ чаще имели эмоциогенный тип нарушений пищевого поведения, для которых потребление пищи выступает одним из инструментов управления эмоциональным состоянием. Результаты согласуются со сведениями из научной литературы, из которых следует, что самые высокие значения по шкалам «невротической триады», в первую очередь по шкале ипохондрии, были свойственны мужчинам с абдоминальным типом распределения жировой ткани [28].

Прием пищи приносит не только целую гамму положительных эмоций - расслабление, радость, спокойствие, но и лишние калории, которые, в свою очередь, приводят к набору массы тела и изменению фигуры [6]. У мужчин с избыточной массой тела, ожирением и АТРЖ изменение внешнего вида может вносить дополнительный вклад в психологические переживания, усугубляя состояние депрессии, в то время как мужчины с ПТРж более довольны своим внешним видом и в большей степени способны самостоятельно регулировать свое состояние [28]. Полученные результаты позволяют говорить, что у мужчин с ПТРЖ ожирение в большей степени ассоциировано с перееданием, ассоциированным с высоким эмоциональным статусом и тревогой, а у мужчин с АТРЖ переедание носит гедонический характер, когда удовольствие от еды становится высшим благом и смыслом жизни.

Возникает вопрос, какие возможные механизмы лежат в основе ассоциации разных типов распределения жировой ткани с определенными типами нарушений пищевого поведения. Результаты исследований, касающихся вопросов возможной связи между уровнем ключевых медиаторов энергетического (инсулина, лептина) и особенностями пищевого поведения противоречивы. Тем не менее большинство авторов признают участие лептина и инсулина в регуляции пищевого поведения $[5,13]$. В проведенном исследовании было показано, что у мужчин с АТРЖ по сравнению с мужчинами с ПТРЖ выше уровни инсулина и лептина в сыворотке крови. Лептин, анорексигенный гормон жировой ткани, участвует в регуляции веса тела и аппетита, ингибируя по механизму отрицательной обратной связи синтез гипоталамического нейропептида $Y$, что приводит к повышению тонуса симпатической нервной системы и расходу энергии $[13,22]$. Повышение уровня лептина при ожирении, особенно при АТРЖ, свидетельствует о формировании лептинорезистентности и нарушениях механизмов его влияния на энергетический обмен $[21,22]$. При гиперинсулинемии гематоэнцефалический барьер становится проницаемым для триптофана, что ведет к усилению синтеза серотонина, участвующего в формировании насыщения, полноценного сна и созданию эмоционального комфорта [29]. Однако у мужчин с АТРЖ на фоне гиперинсулинемии в еще большей степени увеличивается инсулинорезистентность, а в этих условиях отсутствуют необходимые условия 
для достаточного проникновения триптофана через гематоэнцефалический барьер и повышения синтеза серотонина, что влечет за собой отсутствие насыщения и усиливает экстернальную компоненту нарушений пищевого поведения.

Выявленное у обследованных мужчин от группы 1 к группе 4 последовательное повышение содержания в сыворотке крови лептина и понижение - адипонектина, гормона жировой ткани с антиапоптотическими, антифибротическими, противовоспалительными эффектами, повышающего чувствительность клеток к инсулину, привело к резкому повышению величины отношения содержания лептина к адипонектину - в 2,0, 3,2 и 7,8 раза в группах 2, 3 и 4 относительно группы 1. В настоящее время величина этого отношения рассматривается как маркер раннего выявления метаболических нарушений при ожирении, маркер воспаления в жировой ткани [23], атерогенный индекс при сосудистых заболеваниях [24], который коррелирует с инсулинорезистентностью лучше, чем лептин и адипонектин отдельно. Полученные в исследовании результаты свидетельствуют, что у мужчин с ПТРЖ указанные нарушения выражены в наименьшей степени относительно мужчин с АТРЖ.

Опираясь на полученные результаты, можно говорить о том, что конституциональные факторы и гормонально-адипокиновые механизмы определяют не только особенности топографии жировой ткани, но также психоэмоциональный статус и различные типы нарушений ПП, характерные для патогенетически разных вариантов ожирения: с ПТРЖ и АТРЖ. Особенности взаимоотношений психоэмоционального состояния и ПП с разными типами распределения жировой ткани у мужчин необходимо учитывать при разработке индивидуальных программ коррекции веса.

\section{Ограничения исследования}

Ограничением исследования является малый объем выборки.

\section{ЗАКЛЮЧЕНИЕ}

В ранее проведенных исследованиях было показано, что ожирение во многих случаях сопровождается дислипидемиями и гипергликемией, гиперинсулинемией и инсулинорезистентностью, гиперлептинемией, гипоадипокинемией относительно лиц с нормальной массой тела, что определяет его роль как фактора риска развития неинфекционных заболеваний. В настоящее время особое внимание уделяют генетической и гормонально-метаболической гетерогенности ожирения, однако нерешенными остаются вопросы соотнесения типов распределения жировой ткани и, соответственно, типов ожирения с гормонально-метаболическими, адипокиновыми и психофизиологическими характеристиками организма, особенно с учетом гендерных особенностей. В работе показано, что топография распределения жировой ткани у мужчин ассоциирована с психофизиологическими, метаболическими и гормонально-адипокиновыми особенностями, лежащими в основе развития первичного ожирения алиментарно-конституциональной природы.

\section{ДОПОЛНИТЕЛЬНАЯ ИНФОРМАЦИЯ}

Источник финансирования. Исследование проведено в рамках выполнения государственного задания учреждения.

Конфликт интересов. Авторы декларируют отсутствие явных и потенциальных конфликтов интересов, связанных с публикацией настоящей статьи.

Участие авторов. Мохова И.Г. - сбор материала, обработка материала, анализ полученных данных; Пинхасов Б.Б. - разработка дизайна исследования, анализ полученных данных, написание текста; Шилина Н.И. - сбор материала, обработка материала; Янковская С.В. - сбор материала, обработка материала; Селятицкая В.Г. - разработка концепции исследования, критическая интерпретация результатов, написание текста. Все авторы внесли значимый вклад в проведение исследования и подготовку статьи, прочли и одобрили финальную версию статьи перед публикацией.

\section{СПИСОК ЛИТЕРАТУРЫ | REFERENCES}

1. Blüher M. Obesity: global epidemiology and pathogenesis. Nat Rev Endocrinol. 2019;15(5):288-298. doi: https://doi.org/10.1038/s41574-019-0176-8

2. Kelly T, Yang W, Chen C, et al. Global burden of obesity in 2005 and projections to 2030. Int J Obes (Lond). 2008;32(9):1431-1437. doi: https://doi.org/10.1038/ijo.2008.102

3. Zalesin KC, Franklin BA, Miller WM, et al. Impact of obesity on cardiovascular disease. Endocrinol Metabolism Clin North Am. 2008;37(3):663-684. doi: https://doi.org/10.1016/j.ecl.2008.06.004

4. Garvey WT. Clinical Definition of Overweight and Obesity. In: Gonzalez-Campoy J, Hurley D, Garvey WT, editors. Bariatric Endocrinology. Springer: Cham; 2019. p. 121-143. doi: https://doi.org/10.1007/978-3-319-95655-8_7

5. Волкова Г.Е., Романцова Т.И., Вознесенская Т.Г., Роик О.В. Пищевое поведение у пациентов с ожирением // Ожирение и метаболизм. 2007. - T. 4. - № 2. - C. 17-21. [Volkova GE, Romantsova TI, Voznesenskaya TG, Roik OV. Pishchevoe povedenie u patsientov s ozhireniem. Obesity and metabolism. 2007;4(2):17-21. (In Russ.)] doi: https://doi.org/10.14341/2071-8713-5158

6. Исаченкова О.А. Пищевое поведение как важный фактор развития ожирения и коморбидных с ним заболеваний // Ожирение и метаболизм. - 2015. - Т. 12. - № 4. - C. 14-17. [ssachenkova OA. Eating behavior as an important factor in the development of obesity and comorbid diseases. Obesity and metabolism. 2015;12(4):14-17. (In Russ.)] doi: https://doi.org/10.14341/omet2015414-17
7. Lee MJ, Wu Y, Fried SK. Adipose tissue heterogeneity: implication of depot differences in adipose tissue for obesity complications. Mol Aspects Med. 2013;34(1):1-11. doi: https://doi.org/10.1016/..mam.2012.10.001

8. Feijoo-Bandin S, Rodriguez-Penas D, Garcia-Rua V, et al. Adipokines at the cardiovascular system: role in health and disease. SM JEndocrinol Metab. 2016;2(1):1009-1016. doi: https://doi.org/10.2478/immun-2013-0004

9. Шляхто Е.В., Недогода С.В., Конради А.О., и др. Концепция новых национальных клинических рекомендаций по ожирению // Российский кардиологический журнал. - 2016. T. 21. — № 4. - C. 7-13. [Shlyakhto EV, Nedogoda SV, Konradi $\mathrm{AO}$, et al. The concept of novel national clinical guidelines on obesity. Russian journal of cardiology. 2016;21(4):7-13. (In Russ.)] doi: https://doi.org/ 10.15829/1560-4071-2016-4-7-13

10. Dobson R, Burgess MI, Sprung VS, et al. Metabolically healthy and unhealthy obesity: differential effects on myocardial function according to metabolic syndrome, rather than obesity. Int J Obes (Lond). 2016;40(1):153-161. doi: https://doi.org/10.1038/ijo.2015.151

11. Бородкина Д.А., Груздева О.В., Квиткова Л.В. Барабаш О.Л. Распределение жировых отложений: разгадка кажущегося парадокса ожирения в кардиологии? // Ожирение и метаболизм. 2017. - T. 14. - № 2. - C. 3-8. [Borodkina DA, Gruzdeva OV, Kvitkova LV, Barbarash OL. Body fat distribution: the answer to the apparent paradox of obesity in cardiology? Obesity and metabolism. 2017;14(2):3-8. (In Russ.)] doi: https://doi.org/10.14341/omet201723-8 
12. Freedland ES. Role of a critical visceral adipose tissue threshold (CVATT) in metabolic syndrome: implications for controlling dietary carbohydrates: a review. Nutr Metab (Lond). 2004;1:12 doi: https://doi.org/10.1186/1743-7075-1-12

13. Аметов А.С., Абдулкадирова Ф.Р., Покровская Р.А., и др. Ожирение: роль лептина // Лечение и профилактика. - 2014. — № 2. C. 86-91. [Ametov AS, Abdulkadirova FR, Pokrovskaya RA., et al. The obesity: the role of leptin. Disease treatment and prevention. 2014:(2):86-91. (In Russ.)]

14. Han TS, Lean MEJ. A clinical perspective of obesity, metabolic syndrome and cardiovascular disease. JRSM Cardiovasc Dis. 2016;5:2048004016633371 doi: https://doi.org/10.1177/2048004016633371

15. Grundy SM. Metabolic syndrome update. Trends Cardiovasc Med. 2016;26(4):364-373. doi: https://doi.org/10.1016/j.tcm.2015.10.004

16. Heitmann BL, Frederiksen P. Thigh circumference and risk of heart disease and premature death: prospective cohort study. BMJ. 2009:339:b3292. doi: https://doi.org/10.1136/bmj.b3292

17. van Strein T, Frijtere JER, Bergere GPA, Defares PB. The Dutch eating behavior questionnaire (DEBQ) for assessment of restrained emotional and external eating behavior. Int J Eat Disord. 1986;5(2):295-315. doi: https://doi.org/10.1002/1098108X(198602)5:2<295::AID-EAT2260050209>3.0.CO;2-T

18. Zigmond AS, Snaith RP. The Hospital Anxiety and Depression scale. Acta Psychiatr Scand. 1983;67(6):361-370. doi: https://doi.org/10.1111/j.1600-0447.1983.tb09716.x

19. Малкина-Пых И.Г. Терапия пищевого поведения. Справочник практического психолога. - М.: Эксмо; 2007. [Malkina-Pykh IG. Terapiya pishchevogo povedeniya. Spravochnik prakticheskogo psikhologa. Moscow: Eksmo, 2007. (In Russ.)]

20. Пинхасов Б.Б., Селятицкая В.Г., Карапетян А.Р., Лутов Ю.П. Ассоциация возрастного ожирения с метаболическим синдромом у мужчин // Успехи геронтологии. - 2016. - Т. 29. № 1. - C. 86-92. [Pinkhasov BB, Selyatitskaya VG, Karapetyan AR, Lutov YP. Association of aging-related obesity and metabolic syndrome in men. Adv Gerontol. 2016;6(3):224-230. (In Russ.)] doi: https://doi.org/10.1134/\$2079057016030085

21. Moehlecke M, Canani LH, Silva LOJE, et al. Determinants of body weight regulation in humans. Arch Endocrinol Metab. 2016;60(2):152-162. doi: https://doi.org/10.1590/2359-3997000000129

22. Cui H, López M, Rahmouni K. The cellular and molecular bases of leptin and ghrelin resistance in obesity. Nat Rev Endocrinol. 2017;13(6):338-351. doi: https://doi.org/10.1038/nrendo.2016.222
23. Larsen MA, Isaksen VT, Moen OS, et al. Leptin to adiponectin ratio a surrogate biomarker for early detection of metabolic disturbances in obesity. Nutr Metab Cardiovasc Dis. 2018;28(11):1114-1121. doi: https://doi.org/10.1016/j.numecd.2018.06.020

24. Nisar MK, Afaq E, Afaq H, et al. Leptin to Adiponectin ratio as Atherogenic Index in Ischemic Heart Disease Patients. Journal of Islamabad Medical \& Dental College. 2018;7(4):285-290 doi: https://doi.org/10.35787/jimdc.v7i4.265

25. Wang T, Ma X, Peng D, et al. Effects of obesity related genetic variations on visceral and subcutaneous fat distribution in a Chinese population. Sci Rep. 2016;6:20691 doi https://doi.org/10.1038/srep20691

26. Brune JE, Kern M, Kunath A, et al. Fat depot-specific expression of HOXC9 and HOXC10 may contribute to adverse fat distribution and related metabolic traits. Obesity (Silver Spring). 2016;24(1):51-59. doi: https://doi.org/10.1002/oby.21317

27. Pinkhasov BB, Selyatitskaya VG, Karapetyan AR, Astrakhantseva EL. Metabolic syndrome in men and women with upper or lower types of body fat distribution. Health. 2012;4(12A):1381-1389. doi: https://doi.org/10.4236/health.2012.412A200

28. Кодочигова А.И., Мартынова А.Г., Киричук В.Ф. Психологические особенности личности в зависимости от типа распределения жировой ткани у клинически здоровых лиц // Кардиоваскулярная терапия и профилактика. - 2005. - Т. 4. - № 2. - C. 84-89. [Kodochigova Al, Martynova AG, KirichukVF. Psychological personality traits in healthy people with various types of fat tissue distribution. Cardiovascular therapy and prevention. 2005;4(2):84-89. (In Russ.)]

29. Wurtman RJ, Wurtman JJ. Brain serotonin, carbohydrate-craving obesity and depression. Obes Res. 1995;3 Suppl 4:477S-480S doi: https://doi.org/10.1002/j.1550-8528.1995.tb00215.x

30. Соболева Н.П., Руднев С.Г., Николаев Д.В., и др. Биоимпедансный скрининг населения России в центрах здоровья распространенность избыточной массы тела и ожирения // Российский медицинский журнал. - 2014. - Т. 20. — № 4. C. 4-13. [Soboleva NP, Rudnev SG, Nikolayev DV, et al. The bioimpedance screening of population in health centers: prevalence of surplus body mass and obesity. Russian medical journal. 2014;20(4):4-13. (In Russ.)]

31. Герасимов А. Н. Медицинская статистика: учебное пособие. М. - Изд. Медицинское информационное агентство. - 2007. [Gerasimov AN. Medical statistics: study guide. M. Publ. Medical Information Agency. (In Russ.)]

\section{ИНФОРМАЦИЯ ОБ АВТОРАХ [AUTHORS INFO]:}

*Мохова Ираида Георгиевна, аспирант [Iraida G. Mokhova, MD, postgraduate student], Россия, 630060, Новосибирск, улица Тимакова, д. 2 [address: 2 Timakova street, 630117 Novosibirsk, Russia]; ORCID: https://orcid.org/0000-0001-6895-2751; eLibrary SPIN: 1335-2786; e-mail: igmokhova@gmail.com

Пинхасов Борис Борисович, д.М.н. [Boris B. Pinkhasov, MD, PhD]; ORCID: https://orcid.org/0000-0002-4579-425X; eLibrary SPIN: 4848-4370; e-mail: pin@centercem.ru

Шилина Надежда Игоревна, аспирант [Nadejda I. Shilina, postgraduate student];

ORCID: https://orcid.org/0000-0002-4288-4479; eLibrary SPIN: 8868-9059; e-mail: n.kutuzova@ngs.ru

Янковская Светлана Валерьевна, аспирант [Svetlana V. Yankovskaya, postgraduate student];

ORCID: https://orcid.org/0000-0001-8486-3185; eLibrary SPIN: 6478-6922; e-mail: 179324865@list.ru

Селятицкая Вера Георгиевна, д.б.н., профессор [Vera G. Selyatitskaya, MD, PhD in biology, Professor]; ORCID: https://orcid.org/0000-0003-4534-7289; eLibrary SPIN: 9992-0023; e-mail: ccem@centercem.ru

*Автор, ответственный за переписку / Corresponding author.

\section{цИТИРОВАТЬ:}

Мохова И.Г., Пинхасов Б.Б., Шилина Н.И., Янковская С.В., Селятицкая В.Г. Особенности психоэмоционального состояния, пищевого поведения и показателей гормонально-адипокиновой регуляции метаболизма у мужчин с подкожным и абдоминальным типами распределения жира // Ожирение и метаболизм. - 2020. - Т. 17. - №2. - С. 156-163. doi: https://doi.org/10.14341/omet12100

\section{TO CITE THIS ARTICLE:}

Mokhova IG, Pinkhasov BB, Shilina NI, Yankovskaya SV, Selyatitskaya VG. The features of psychological state, eating behavior, hormonal and adipokine regulation of metabolism in men with subcutaneous and abdominal fat distribution. Obesity and metabolism. 2020;17(2):156-163. doi: https://doi.org/10.14341/omet12100 